\title{
Right heart failure due to arteriovenous fistula after lumbar spine surgery
}

Seong Soon Kwon ${ }^{1}$, Byoung-Won Park ${ }^{1}$, Min-Ho Lee ${ }^{1}$, Dong Erk Goo ${ }^{2}$, and Bo Da Nam²

${ }^{1}$ Division of Cardiology, Department of Internal Medicine, ${ }^{2}$ Department of Radiology, Soonchunhyang University Seoul Hospital, Seoul, Korea

Received: December 10, 2018

Revised : January 18, 2019

Accepted: January 23, 2019

Correspondence to

Byoung-Won Park, M.D.

Tel: +82-2-709-9215

Fax: +82-2-709-9083

E-mail: s78138@schmc.ac.kr

Figure 1. (A, B) Simple X-ray shows right sided large pleural effusion and posterolateral interbody fusion state of 4-5 lumbar spine. (C) Schematic results of right heart catheterization shows oxygen step up most high in inferior vena cava (IVC). (D, E) Aorta computed tomography angiography shows right common iliac arteriovenous fistula (arrow) with marked enlargement of hepatic IVC, right common/external/internal iliac veins. Ao, aorta; SVC, superior vena cava; AP, pulmonary artery; LA, left atrium; PCWP, pulmonary capillary wedge pressure; RA, right atrium; RV, right ventricle; $\mathrm{LV}$, left ventricle.
A 62-year-old woman was referred to our hospital with recurrent right pleural effusion after lumbar spine surgery 18 months earlier. Immediately after the surgery, she received medical treat- ment for pneumonia and right heart failure (Fig. $1 \mathrm{~A}$ and $1 \mathrm{~B}$ ). Her symptoms persisted despite tricuspid valve annuloplasty for tricuspid regurgitation. She received thoracentesis ( 1 to $2 \mathrm{~L}$ per
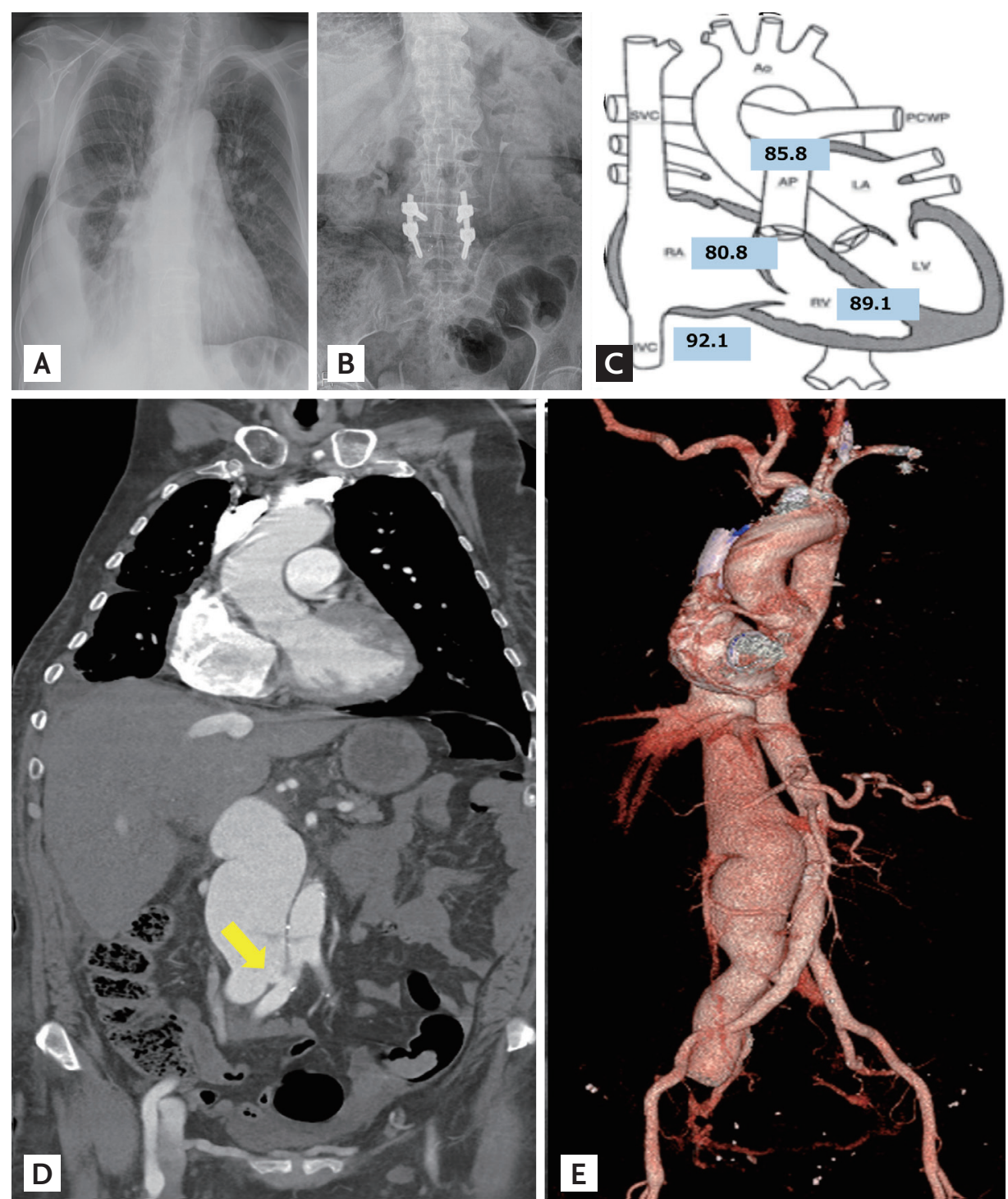

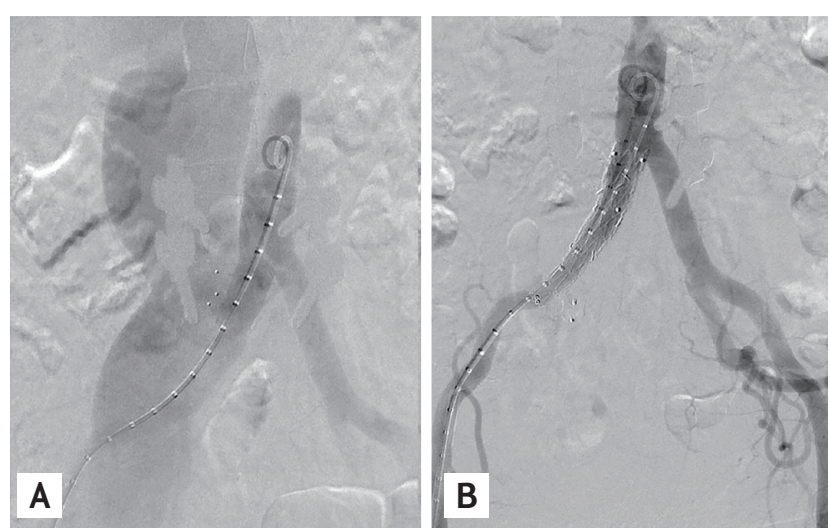

Figure 2. Abdominal aortography. (A) Abdominal aortogram shows arteriovenous fistula in the right common iliac artery to inferior vena cava. (B) Completely sealed arteriovenous fistula by $14 \times 14 \mathrm{~mm}$ and $16 \times 82 \mathrm{~mm}$ stent graft placement in the right common iliac artery after internal iliac arterial embolization using $10 \mathrm{~mm}$ Amplaz plug (AGA Medical Corp.).

week). An echocardiogram showed a dilated right heart, increased pulmonary arterial pressure (maximum pressure gradient: $59 \mathrm{mmHg}$ ) and normal ejection fraction. At first, we suspected chronic thromboembolic pulmonary hypertension and proceeded with the computed tomography (CT) pulmonary angiogram, but results were negative. To identify the cause of pulmonary hypertension, we performed right heart catheterization. Pulmonary capillary wedge pressure was $23 \mathrm{mmHg}$ and the ratio of pulmonary-systemic blood flow was 2.66, indicating the presence of left to right shunt. The highest oxygen step-up was confirmed in the inferior vena cava (IVC) (Fig. 1C). Transesophageal echocardiography and heart magnetic resonance were negative for intracardiac shunt. During auscultation of lung sounds 12 days post-admission, we found a holosystolic murmur in her back. She underwent aorta CT angiography for detection of an extracardiac shunt. An arteriovenous fistula between the right common iliac artery and IVC was found (Fig. $1 \mathrm{D}$ and $1 \mathrm{E}$ ). A $16 \times 82 \mathrm{~mm}$ sized stent graft was deployed across the fistula (Fig. 2). After the procedure, her symptoms and right pleural effusion did not relapse.

In our case, arteriovenous fistulas may be formed immediately when the laceration includes both arterial and venous tearing during lumbar surgery. Multiple tests and time were needed to find the cause of right heart failure. Had we detected her abnormal lung sounds earlier, the diagnosis would have been faster, without unnecessary tests. This case demonstrates that the integration of clinical history and physical exam results in differential diagnosis is crucial for timely diagnosis.

Written informed consent was obtained from the patient who participated in this study.

\section{Conflict of interest}

No potential conflict of interest relevant to this article was reported.

\section{Acknowledgments}

This paper was supported by Soonchunhyang University Research Fund in 2019. 